\title{
The extended hard X-ray emission from the Vela Plerion
}

\author{
V. Mangano ${ }^{1}$, E. Massaro ${ }^{2}$, F. Bocchino ${ }^{3}$, T. Mineo ${ }^{1}$, and G. Cusumano ${ }^{1}$ \\ 1 Istituto di Astrofisica Spaziale e Fisica Cosmica - Sezione di Palermo, IASF-CNR, via Ugo La Malfa 153, \\ 90146 Palermo, Italy \\ e-mail: vanessa@pa.iasf.cnr.it \\ 2 Dipartimento di Fisica, Università La Sapienza, Piazzale A. Moro 2, 00185 Roma, Italy \\ 3 INAF-Osservatorio Astronomico di Palermo, Piazza dei Normanni 1, 90100 Palermo, Italy
}

Received 15 November 2004 / Accepted 6 February 2005

\begin{abstract}
We present the results of a broad band (3-200 keV) spectral analysis of BeppoSAX and XMM-Newton observations of the Vela plerion. The hard X-ray $(>15 \mathrm{keV})$ emission is found to be extended over a region of $12^{\prime}-15^{\prime}$ radius, corresponding to a size of about $1.0-1.3 \mathrm{pc}$ for a source distance of $290 \mathrm{pc}$. A single power law does not give an acceptable fit while a broken power law or a log-parabolic law nicely fit the data. The former spectral model has the photon index $\Gamma_{1}=1.66 \pm 0.01$ for energies lower than the break value equal to $12.5 \mathrm{keV}$ and the index $\Gamma_{2}=2.01 \pm 0.05$ up to about $200 \mathrm{keV}$. The total X-ray luminosity of the Vela plerion is $L_{\mathrm{X}}=5.5 \times 10^{33} \mathrm{erg} \mathrm{s}^{-1}$, which implies a conversion factor of the spin-down power of $\sim 10^{-3}$.
\end{abstract}

Key words. stars: pulsars: general - shock waves - ISM: supernova remnants - ISM: individual object: Vela PWN X-rays: general

\section{Introduction}

The structure and evolution of filled-centre SNRs, also known as plerions according the original definition by Weiler \& Panagia (1978), is a rich subject that involves many physical processes from hydrodynamics to high energy particle acceleration and radiation. Plerions are powered by young pulsars which inject in the remnant a relativistic wind containing high energy particles and a magnetic field. The physical mechanisms that convert the original Pointing-dominated energy flux into particle energy is not completely understood. Furthermore, there is evidence that particles are accelerated inside the plerion at a relatively large distance from the pulsar but the basic mechanism is not entirely clear. Plerion emission is observed across the entire electromagnetic spectrum, from radio waves to the most energetic $\gamma$-rays. However, in some frequency ranges, only little data are available: for example, in the hard $\mathrm{X} /$ soft $\gamma$-rays very little is known about the spectra of plerions.

The Vela SNR is one of the nearest plerions and its structure can be studied in detail. Its distance is estimated to be about 300 pc (Caraveo et al. 2001; Dodson et al. 2003), and therefore an angular size of $1^{\prime}$ corresponds to $0.09 \mathrm{pc}\left(2.7 \times 10^{17} \mathrm{~cm}\right)$. The best Chandra X-ray images show a compact nebula with a jet-counter jet originating from the pulsar and a double bow structure (Helfand et al. 2001; Pavlov et al. 2001a). Kargaltsev \& Pavlov (2003) presented maps of the $2-10 \mathrm{keV}$ photon in$\operatorname{dex}\left(F\left(E_{\gamma}\right) \propto E_{\gamma}^{-\Gamma}\right)$ with resolutions of 2 .'5 and $10^{\prime \prime}$ which show that the spectrum is harder $(\Gamma \simeq 1.2-1.4)$ in the near surroundings of the pulsar and becomes softer $(\Gamma \simeq 1.6-1.8)$ at distances of $2^{\prime}-3^{\prime}$. A more detailed study of the inner Vela Pulsar Wind Nebula (PWN), based on 13 X-ray images, spread over a time interval of 2.5 years, has been performed by Pavlov et al. (2003). In particular, these authors report the presence of bright blobs moving away along the outer jet and fading on time scales of weeks. Photon indices in the band 1.0-8.0 keV derived for the various structures are generally between 1.2 and 1.5 .

Results of a spectral analysis of XMM data have been presented by Mori et al. (2004): their spectral analysis of PN data, extracted from a circular region around the pulsar position within a radius of $1^{\prime}$, gave a photon index of $1.64 \pm 0.08$.

The present knowledge about the hard X-ray emission from the Vela SNR is rather poor. The most recent data are those obtained in the energy range $60-400 \mathrm{keV}$ with OSSECGRO (FOV $3.8 \times 11^{\circ} .4$ ) (De Jager et al. 1996): a single power law best fit indicated a quite hard spectrum with the rather uncertain photon index of $1.6 \pm 0.5$. It was found consistent with the previous $(2-25 \mathrm{keV})$ data obtained with the Birmingham Spacelab 2 coded mask detector (Willmore et al. 1992), that gave $\Gamma=1.74 \pm 0.08$ inside a circle of radius $6^{\prime}-7^{\prime}$. Furthermore these authors found evidence for a more extended emission with a much softer spectrum.

On the basis of a new model for the synchrotron compact nebular emission, Sefako \& De Jager (2003) estimated for the Vela plerion the acceptable ranges of the two main PWN parameters: the wind magnetization $\sigma$ (Kennel \& Coroniti 1984), whose value is expected in the range $0.05-0.5$, and the pair production multiplicity $M$, in the interval between 300 and 700. These authors used the power law spectrum derived 


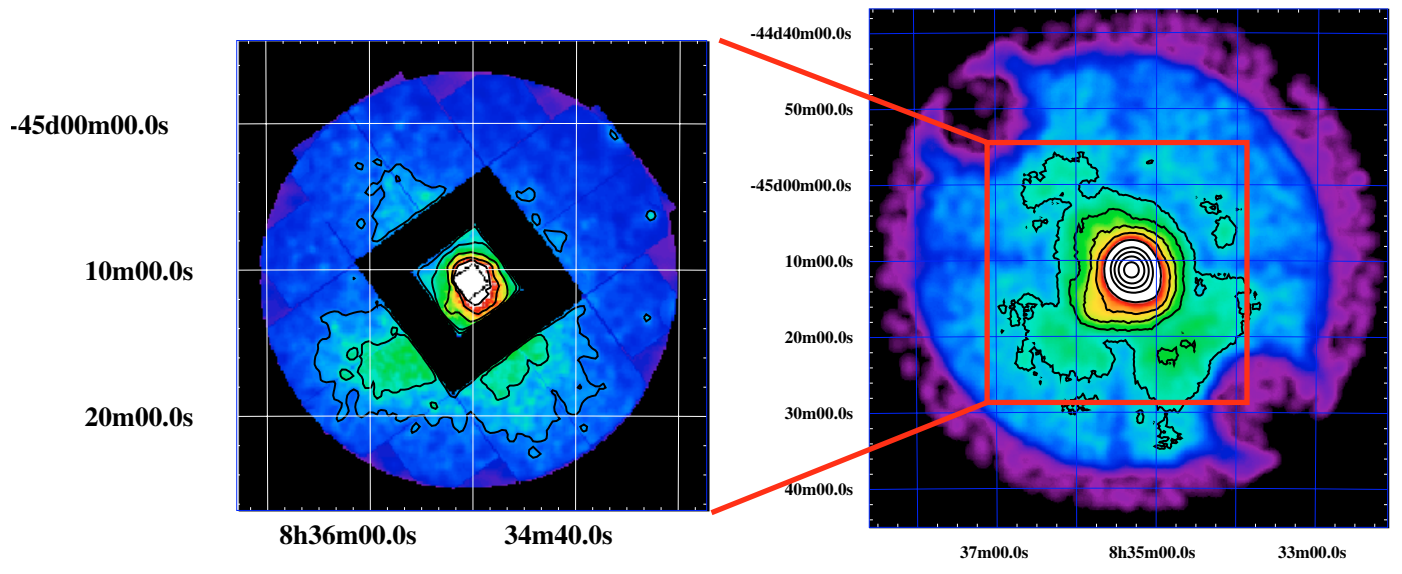

Fig. 1. Left panel: XMM-MOS1 image of the Vela PWN in the $3-10 \mathrm{keV}$ band; contour levels are at $5 \times 10^{-4}, 1 \times 10^{-3}, 2 \times 10^{-3}$ and $8 \times 10^{-3}$ times the brightest point. Right panel: MECS image of the source; outer contour levels are at $3 \times 10^{-3}, 7 \times 10^{-3}, 13 \times 20^{-3}$ and $33 \times 10^{-3}$ times the brightest point. Note the more extended emission in the south-west and south-east regions.

from OSSE data and assumed that the Spectral Energy Distribution (SED) peaks in the $\mathrm{MeV}$ range.

In this paper we present the results of the analysis of BeppoSAX data of the Vela Plerion in the energy range 3-200 keV combined with the imaging and spectral capabilities of XMM-Newton. We derived information on the size of the source emission above $10 \mathrm{keV}$ and obtained an accurate evaluation of the spectral parameters, useful to measure the total energy output from this plerion and for a more accurate comparison with models of nebular emission.

\section{BeppoSAX and XMM-Newton observations and data reduction}

The XMM-Newton observations were performed on 1 and 2 December 2000 with an exposure of 38 and $57 \mathrm{ks}$, respectively. We reduced the EPIC data with the XMM Science Analysis System (SAS) version 6.0 and performed standard screening of the EPIC data. The 2 December 2000 observation suffered high background contamination and required a careful rejection of time intervals with soft proton flares; to asses the degree of soft proton contamination we used the diagnostic defined by De Luca \& Molendi (2004). Because of this additional screening the effective exposure in the 2 December 2000 observation was reduced to $47 \mathrm{ks}$. In both observations MOS1 was operated in Large Window mode, where the inner active region of the MOS array is the central square of 5.5 side (see Fig. 1, left panel). MOS2 was working in Small Window mode with an active central square of 1.'8 side only and was not included in the analysis of the wider central plerion emission. PN was working in Small Window mode but the target was not centred in the active square region and therefore PN data were not included in our analysis.

Diffuse and asymmetric emission is visible in the image of Fig. 1 (left panel) beyond $8^{\prime}$ mostly in South-East and SouthWest directions. Comparing the X-ray contour with the radio images recently obtained by Dodson et al. (2003) we note that the structure of the diffuse emission is correlated with that observed at radio frequencies. We extracted spectra from circular regions centred on the source with a radius of $0.5,1.0,1.5$ and 2.0 (therefore including the central source) and, to investigate better the spectral changes moving outward in the nebula, also from annular regions centred at the source with radii $0.5-1.0,1.0-1.5,1.5-2{ }^{\prime} .0$ and $8^{\prime}-12^{\prime}$ (outside the gap). We also extracted spectra from two circles of $2^{\prime}$ radius in the SouthEast (RA: 08h35m52s, Dec: $-45 \mathrm{~d} 16 \mathrm{~m} 26 \mathrm{~s}$ ) and South-West (RA: 08h34m58s, Dec: $-45 \mathrm{~d} 17 \mathrm{~m} 04 \mathrm{~s}$ ) regions where, according to Fig. 1, diffuse emission is visible. Spectra and effective area corresponding to the two observations were summed using the tasks mathpha and addarf from the HEASOFT 5.3.1 package.

The spectrum of the background for the MOS1 was extracted from a set of 10 public galactic plane observations at $l=310^{\circ}-312^{\circ}$ from which we have removed the detected sources, and, for comparison, from closed datasets (Marty et al. 2002) containing only the instrumental and particle components of the background. Results obtained using these two different background spectra were always compatible. Vignetting correction was properly applied to both source and background spectra. Spectral response matrices and effective area were calculated for each region via the SAS tasks rmfgen and arfgen, respectively, according to the standard recipe for vignettingcorrected extended sources.

The BeppoSAX observation of the Vela pulsar region was performed on 18-20 November 1997 for a total net exposure of $84 \mathrm{ks}$ in the MECS and $38 \mathrm{ks}$ in the PDS (for details on the BeppoSAX instrumentation see Boella et al. 1997). Standard procedures and selection criteria were applied to the data using the SAXDAS v.2.0.0 package. The image extracted from the MECS (Fig. 1, right panel) presents regions of diffuse emission in the South-East and South-West directions just as in the XMM image. The extended structure of the source requires that a suitable auxiliary matrix must be created to correct for the redistribution due to the instrument point spread function and for the vignetting relevant at distances larger than about 4' from the centre of the FOV (Fiore et al. 1999). A proper response matrix can be generated for the MECS data using the SAXDAS effarea command (for details see Molendi 1998; D'Acri et al. 1998) and the average radial profile of the Vela plerion derived from the XMM-Newton image interpolated with a spline function in 
the gap region and extrapolated with a constant up to $15^{\prime}$. We produced ad-hoc matrices for the circular collecting areas of radii equal to $4^{\prime}, 8^{\prime}, 12^{\prime}$ and $15^{\prime}$. The poor angular resolution of the MECS and the difficulty of producing a good response matrix for annular regions did not allow us to perform a spatial differential study of the X-ray emission, as done for MOS data.

The spectrum of the background for the MECS was extracted from archive blank fields.

PDS data were collected using the standard rocking observation mode with two detectors pointing at the source and the other two in offset directions to measure the background simultaneously. The net count rate in the whole energy band was $1.7 \mathrm{c} / \mathrm{s}$. The PDS field of view, $1.3^{\circ} \mathrm{FWHM}$, is large enough to include the entire plerion emission. It is, however, much narrower than that of OSSE $(3: 8 \times 11: 4 F W H M)$. We also checked that PDS field of view does not contain bright sources that could contaminate the Vela spectrum.

Events with an energy higher than $3 \mathrm{keV}$ were selected for the analysis to exclude the soft thermal contribution from the pulsar (Pavlov et al. 2001b) and from the Vela shell (Bocchino et al. 1999). Effective ranges were $3-10 \mathrm{keV}$ for MECS and MOS and 15-200 keV for PDS. For the same reason we did not consider LECS data in the analysis. Spectral fits were first evaluated separately for the MOS, MECS and PDS data and after, combined fits of MOS-PDS and MECS-PDS data sets were considered.

A relevant point we stress when working with spectra obtained with several instruments is that a proper evaluation of the inter-calibration factors is required. For the BeppoSAX NFIs, the accurate ground and in-flight calibrations were used to establish that the admissible ranges for the factor between MECS and PDS for point sources $\left(f_{M P}\right)$ is $0.77 \leq f_{M P} \leq 0.93$, reduced to $0.86 \pm 0.03$ for sources with a PDS count rate higher than $2 \mathrm{ct} / \mathrm{s}$ (Fiore et al. 1999). For extended sources, as for the Vela plerion, we expect that the best fit estimate of $f_{M P}$ would result in agreement with the above value only when the emission observed by the PDS is fully selected in the MECS image. Moreover, MOS-PDS intercalibration factor is expected in a similar range because the intercalibration analysis between MOS and MECS shows a good agreement between the two instruments (Kirsh 2004). Values of the intercalibration factors well above the expected range can be an indication that MECS flux, for a given collecting area, includes only a fraction of the signal detected by the PDS. Thus it is possible to use this parameter to obtain information on the angular size of the source responsible for the hard X-ray $(>15 \mathrm{keV})$ emission.

The uncertainties reported in the following are at 1 standard deviation for one interesting parameter.

\section{Spectral analysis}

\subsection{Single instrument spectral analysis}

Spectra of each instrument were fitted with a single power-law model. A plot of the MOS and MECS photon index as a function of the extraction radius for all the circular regions centred at the source is shown in Fig. 2 . The value of $\Gamma$ changes with the extraction radius: for a radius $\leq 2^{\prime}$ it is around 1.55 , while

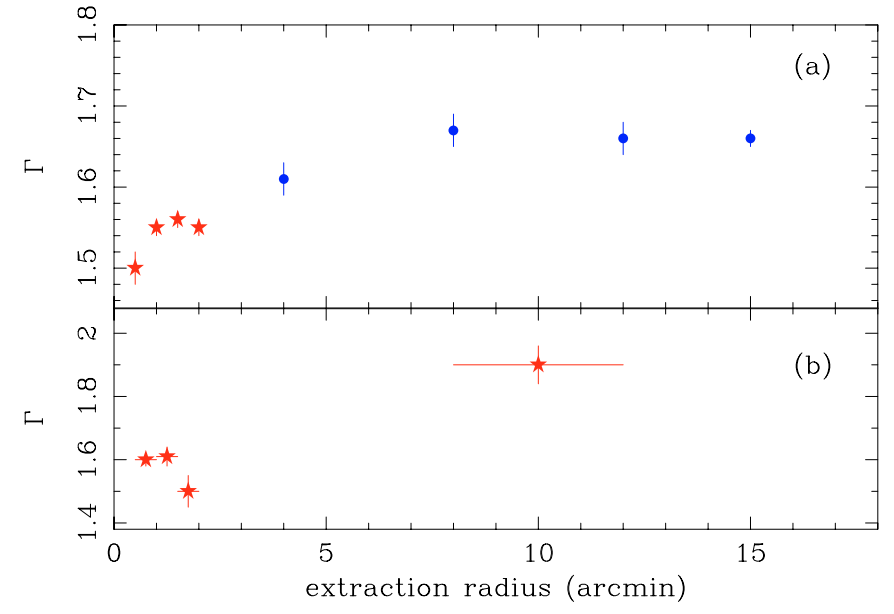

Fig. 2. Panel a): the power law photon index in the $3-10 \mathrm{keV}$ band of MOS (stars) and MECS (filled circles) spectra at varying extraction radius. Panel b): the power law photon indices in the $3-10 \mathrm{keV}$ band of MOS spectra extracted from the annuli listed in Table 1.

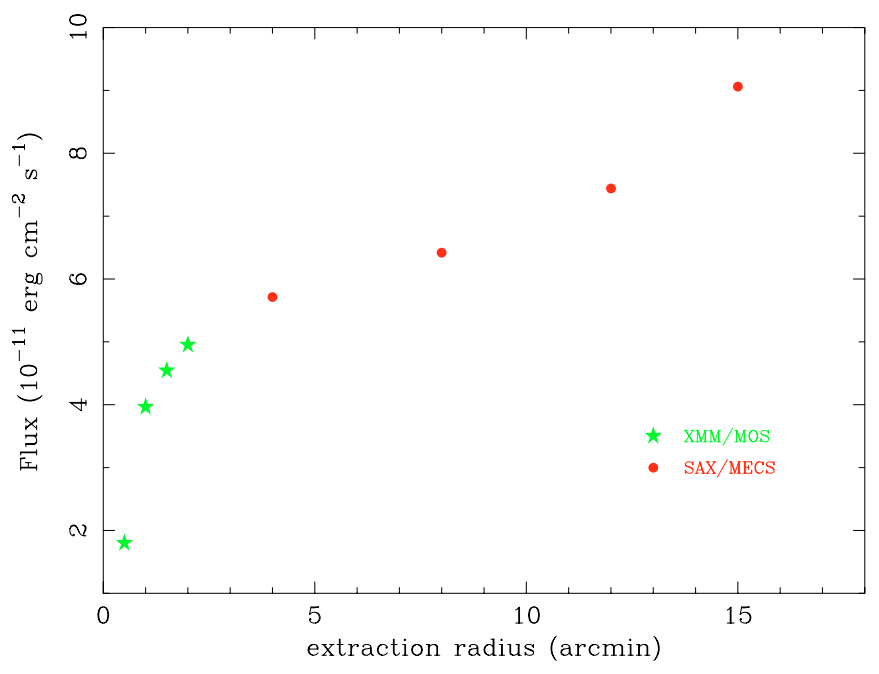

Fig. 3. Vela PWN flux in the $3-10 \mathrm{keV}$ band at varying extraction radius.

for higher radii it increases to 1.67 , in agreement, within the errors, with the value reported by Willmore et al. (1992), indicating a quite mild softening of the spectrum in the outer region of the PWN. Figure 3 shows the fluxes in the $3-10 \mathrm{keV}$ band as a function of the spectra extraction radius. The flux increase within $4^{\prime}$ quantifies the amount of emission originating from the inner region.

The spatially differential spectral analysis confirms that the inner region of the plerion has a flatter spectrum than the outer one. The average spectral index of the three annuli is $1.60 \pm 0.02$ (the third annulus differs from this value only by two standard deviations) to be compared with 1.90 found at distances greater than $8^{\prime}$. The small difference (at about two standard deviations) between the mean index of the three inner annuli and that found for the circular region of 0.5 radius is likely due to the contribution of the inner part of the PWN which is about $40 \%$ of the total as derived from the $3-10 \mathrm{keV}$ flux ratio. 
Table 1. Best fit parameters for a single power law of MOS and MECS. The values given in the first section are for the spatially integrated analysis, while those of the second section are for the MOS annular regions.

\begin{tabular}{cccccc}
\hline \hline & Radius & $\Gamma^{a}$ & $K^{a, b}$ & $F^{a, c}$ & $\chi_{r}^{2}$ (d.o.f.) \\
\hline MOS & $0^{\prime} .5$ & $1.50 \pm 0.02$ & $0.39 \pm 0.01$ & $1.785 \pm 0.009$ & $1.16(253)$ \\
MOS & $1^{\prime}$ & $1.55 \pm 0.01$ & $0.95 \pm 0.02$ & $3.98 \pm 0.01$ & $1.08(324)$ \\
MOS & $1^{\prime} .5$ & $1.56 \pm 0.01$ & $1.09 \pm 0.02$ & $4.49 \pm 0.01$ & $1.11(331)$ \\
MOS & $2^{\prime}$ & $1.55 \pm 0.01$ & $1.18 \pm 0.02$ & $4.95 \pm 0.01$ & $1.07(337)$ \\
\hline MECS & $4^{\prime}$ & $1.61 \pm 0.02$ & $1.50 \pm 0.04$ & $5.66 \pm 0.03$ & $0.96(66)$ \\
MECS & $8^{\prime}$ & $1.67 \pm 0.02$ & $1.88 \pm 0.05$ & $6.39 \pm 0.03$ & $0.93(66)$ \\
MECS & $12^{\prime}$ & $1.66 \pm 0.02$ & $2.17 \pm 0.05$ & $7.50 \pm 0.03$ & $1.02(66)$ \\
MECS & $15^{\prime}$ & $1.66 \pm 0.01$ & $2.62 \pm 0.09$ & $9.06 \pm 0.04$ & $1.02(66)$ \\
\hline PDS & FOV & $2.00 \pm 0.05$ & $6.19 \pm 0.95^{d}$ & $15.9 \pm 0.4^{e}$ & $0.73(16)$ \\
\hline MOS & $0^{\prime} 5-1^{\prime}$ & $1.60 \pm 0.02$ & $0.56 \pm 0.02$ & $2.150 \pm 0.009$ & $1.05(209)$ \\
MOS & $1^{\prime}-1.5$ & $1.61 \pm 0.03$ & $0.14 \pm 0.01$ & $0.528 \pm 0.005$ & $0.88(72)$ \\
MOS & $1^{\prime} .5-2^{\prime}$ & $1.50 \pm 0.05$ & $0.08 \pm 0.01$ & $0.361 \pm 0.004$ & $0.77(49)$ \\
MOS & $8^{\prime}-12^{\prime}$ & $1.90 \pm 0.06$ & $0.51 \pm 0.05$ & $1.165 \pm 0.02$ & $1.13(231)$ \\
\hline
\end{tabular}

${ }^{a}$ Errors correspond to 1 standard deviation for one interesting parameter.

${ }^{b}$ Power law normalisation $K$ is expressed in units of $10^{-2}$ photons $\mathrm{cm}^{-2} \mathrm{~s}^{-1} \mathrm{keV}^{-1}$ at $1 \mathrm{keV}$.

${ }^{c}$ Flux is in units of $10^{-11} \mathrm{erg} \mathrm{cm}^{-2} \mathrm{~s}^{-1}$. It is relative to the $3-10 \mathrm{keV}$ band for the MOS/MECS and to the 20-100 keV band for the PDS. The reported errors are statistical. Systematical errors amount to 5\% for MOS flux values and $10 \%$ for the MECS.

${ }^{d}$ The PDS normalisation is obtained dividing the single power law fit result $(5.2 \pm 0.8)$ by the intercalibration factor $f_{M P}=0.84$ obtained in the combined fit with the MECS spectrum extracted from the circle of $15^{\prime}$ radius.

${ }^{e}$ The PDS flux value is that obtained from the single power law fit $(13.39 \pm 0.34)$ divided by the intercalibration factor $f_{M P}=0.84$.

The spectral index variation in the inner three MOS annuli is in agreement with the Chandra spectral index map presented by Kargaltsev \& Pavlov (2003).

Fitting the PDS spectrum in the energy range $15-200 \mathrm{keV}$ gives a photon index equal to $2.00 \pm 0.05$ with the $\chi_{r}^{2}$ of 0.73 for 16 d.o.f. and a flux of $(15.7 \pm 0.4) \times 10^{-11} \mathrm{erg} \mathrm{cm}^{-2} \mathrm{~s}^{-1}$. This photon index, significantly higher than those found in the lower energy range, indicates that a spectral steepening occurs at higher energies. Moreover, it is higher than the value obtained with OSSE (De Jager et al. 1996), although statistically compatible because of the large uncertainty in their estimate. A summary of the best fit results for the various extraction radii of the imaging instruments and PDS are given in Table 1.

We also investigated the spectral properties in the outer part of the plerion. The XMM spectra extracted from two circular regions having a diameter of $4^{\prime}$ in the South-East and South-West were also fitted with a simple power-law model. We obtained $\Gamma=1.9 \pm 0.1, K=(5.0 \pm 1.7) \times 10^{-4}$ photons $\mathrm{cm}^{-2} \mathrm{~s}^{-1} \mathrm{keV}^{-1}\left(\chi_{r}^{2}=0.94,181\right.$ d.o.f. $)$ in the SE region and $\Gamma=1.9 \pm 0.1, K=(5.5 \pm 1.5) \times 10^{-4}$ photons $\mathrm{cm}^{-2} \mathrm{~s}^{-1} \mathrm{keV}^{-1}\left(\chi_{r}^{2}=1.01,186\right.$ d.o.f.) in the $\mathrm{SW}$ region, respectively. The $3-10 \mathrm{keV}$ band fluxes per unit solid angle from the two regions were $(8.4 \pm 0.3) \times 10^{-7} \mathrm{erg} \mathrm{cm}^{-2} \mathrm{~s}^{-1} \mathrm{sr}^{-1}$ and $(9.6 \pm 0.5) \times 10^{-7} \mathrm{erg} \mathrm{cm}^{-2} \mathrm{~s}^{-1} \mathrm{sr}^{-1}$.

\subsection{Combined instrument spectral analysis}

The finding that the $15-200 \mathrm{keV} \mathrm{X}$-ray photon index is significantly larger than those measured below $10 \mathrm{keV}$ indicates that a single power law is not able to fit the broad band spectrum of the Vela PWN. We therefore performed a spectral analysis combining the PDS data with those of MOS/MECS. The single power-law model was rejected because the $\chi_{r}^{2}$ values of the combined fits were in the range 1.34 (341 d.o.f.) 1.84 (83 d.o.f.). Moreover, PDS residuals show very large deviations with $\chi_{r}^{2}$ always higher than 4 (15 d.o.f.).

Two other models were then considered: a broken power law:

$\begin{array}{ll}F(E)=K(E / 1 \mathrm{keV})^{-\Gamma_{1}} & \text { for } E<E_{\mathrm{b}} \\ F(E)=K E_{\mathrm{b}}^{\left(\Gamma_{2}-\Gamma_{1}\right)}(E / 1 \mathrm{keV})^{-\Gamma_{2}} & \text { for } E>E_{\mathrm{b}}\end{array}$

and a continuous steepening law, characterised by a linear dependence of the spectral slope upon the logarithm of energy:

$F(E)=K(E / 1 \mathrm{keV})^{-(\alpha+\beta \log (E / 1 \mathrm{keV}))}$.

The fits of the broken power law (Eq. (1)) to the spectra for different extraction radii gave acceptable $\chi_{r}^{2}$ for all regions but showed a strong correlation between the intercalibration factor $f_{M P}$ and the break energy $E_{\mathrm{b}}$. To extract reliable values from this model it is necessary to fix one of these two parameters.

The log-parabolic law (Eq. (2)) gave acceptable fits for all extraction radii without evidence of a correlation between the parameters. The best fit values of $f_{M P}$ are outside the expected range with extraction radii lower than $15^{\prime}$ as shown in Fig. 4. This result indicates that to match the PDS signal to the 3-10 keV flux we must extract events in a MECS region with a radius of about $15^{\prime}$.

To further investigate the extension of the region responsible for the emission above $15 \mathrm{keV}$ we fixed $f_{M P}$ to 0.93 (the highest acceptable value) and, fitting the $12^{\prime}$ spectrum with 


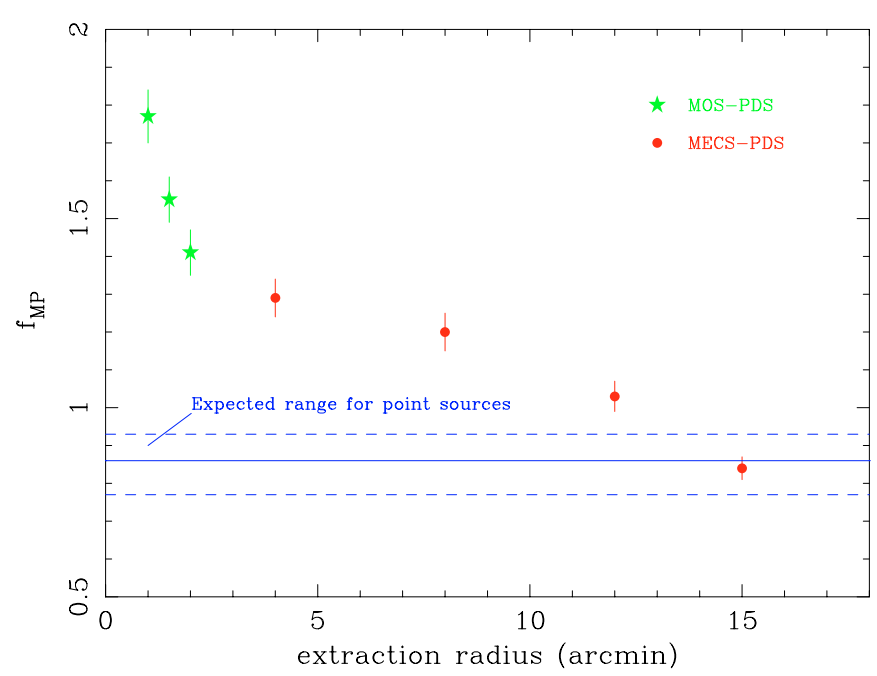

Fig. 4. Best fit values of $f_{M P}$ in the combined log-parabolic fit with the PDS at varying extraction radius.

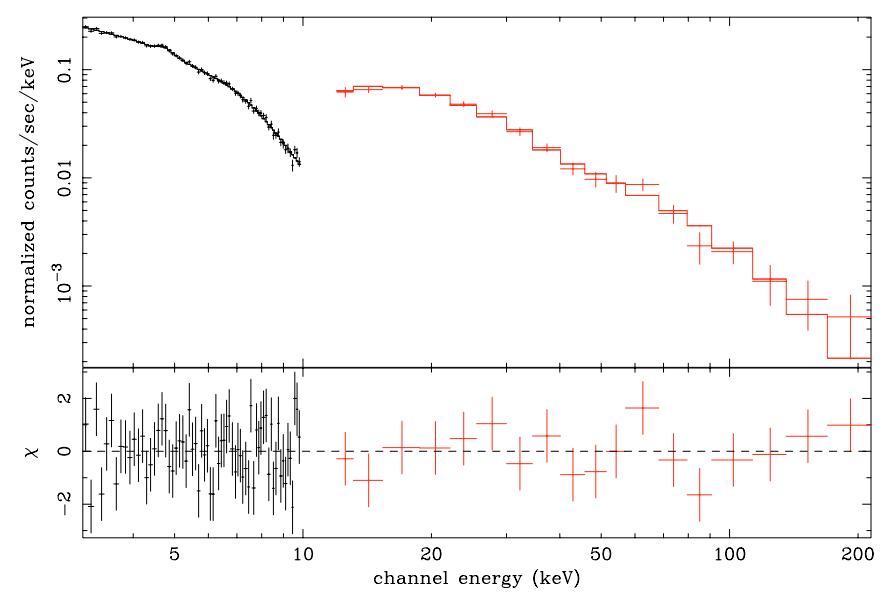

Fig. 5. Best fit of MECS and PDS data with a broken power law model (15' extraction radius for the MECS data). See Table 2 for the best fit parameter values.

Eq. (2), we obtained an acceptable $\chi_{r}^{2}$ of 0.98 (83 d.o.f.) and an acceptable $\chi_{r}^{2}$ relative to PDS data only of 1.09 (15 d.o.f.). However, the same procedure on the $8^{\prime}$ spectrum gave a marginally acceptable fit for the two datasets $\left(\chi_{r}^{2}=1.3\right.$, 83 d.o.f.) but not acceptable for PDS data only $\left(\chi_{r}^{2}=2.90\right.$, 15 d.o.f.). Thus, we are strongly confident that the extension of the hard X-ray emitting region is at least $12^{\prime}$ from the pulsar.

We also fitted the MECS spectrum extracted from a $15^{\prime}$ radius and the PDS spectrum with the broken power-law model, fixing the intercalibration factor to the value obtained in the $\log$-parabolic fit. The fit gave an acceptable $\chi_{r}^{2}$. The spectrum and the best fit model are shown in Fig. 5, and the parameters' values are given in Table 2. Note that the hard branch of the spectrum is fully consistent with the fit of PDS as single instrument (see Table 1).

We then verified if the models used to describe the spectral distribution of the Vela PWN in the $3-200 \mathrm{keV}$ were consistent with the EGRET upper limits reported by Kanbach et al. (1994) for the unpulsed component observed from the region of PSR 0833-45. We extrapolated the two best fit spectra of

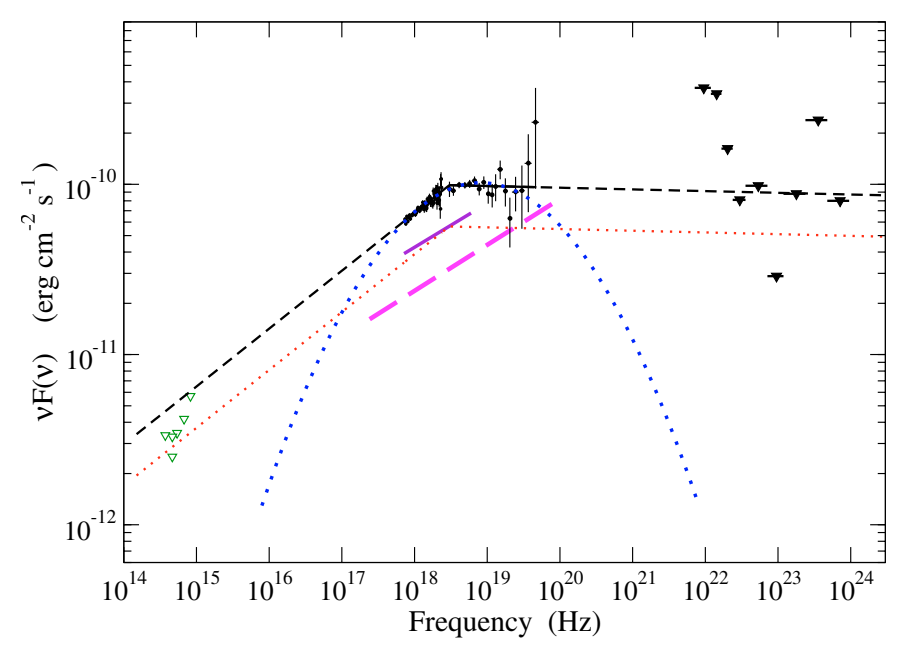

Fig. 6. The optical to X-ray spectral energy distribution of the Vela PWN compared with other literature results. Our SEDs are the broken power law (solid and short-dashed lines) and the log-parabola (dotted line) to which are superposed the deconvolved spectral data. The thick solid line is the spectrum measured by the Birmingham 2 experiment (Willmore et al. 1992) and the dotted lines represent the broken power law SED rescaled to the flux measured by the MECS with an extraction radius of $4^{\prime}$. The thick long dashed line is the broad band spectrum of the compact nebula based on OSSE data (De Jager 1996) and used by by Sefako \& De Jager (2003). Optical upper limits (empty triangles) are taken from Mignani et al. (2003) extrapolated to an area of $10^{4} \operatorname{arcsec}^{2}$ to cover all the inner part of the plerion. The triangles in the $\gamma$-ray band represent EGRET upper limits from Kanbach et al. (1994).

Table 2. Best-fit model parameters of the combined fit MECS-PDS relative to the MECS extraction radius of $15^{\prime}$.

\begin{tabular}{|c|c|}
\hline \multicolumn{2}{|c|}{ Log-parabolic Model } \\
\hline$\alpha$ & $1.36 \pm 0.05$ \\
\hline$\beta$ & $0.21 \pm 0.03$ \\
\hline$K\left(\times 10^{-2}\right)$ & $2.08 \pm 0.10$ \\
\hline$f_{M P}$ & $0.84 \pm 0.03$ \\
\hline$\chi_{r}^{2}$ (d.o.f.) & $0.76(82)$ \\
\hline \multicolumn{2}{|c|}{ Broken power-law Model } \\
\hline$\Gamma_{1}$ & $1.66 \pm 0.01$ \\
\hline$\Gamma_{2}$ & $2.01 \pm 0.05$ \\
\hline$E_{\mathrm{b}}$ & $12.5 \pm 1.5$ \\
\hline$K\left(\times 10^{-2}\right)$ & $2.62 \pm 0.06$ \\
\hline$f_{M P}$ & 0.84 (fixed) \\
\hline$\chi_{r}^{2}$ (d.o.f.) & $0.96(82)$ \\
\hline
\end{tabular}

the combined MECS-PDS data sets $\left(15^{\prime}\right.$ extraction radius for the MECS) in the EGRET range and the results are shown in Fig. 6. While the log-parabolic spectrum is much lower than the $\gamma$-ray upper limits, that derived from the broken power law is only marginally compatible with them. It is possible, however, that the plerion spectrum will show another break and/or a cutoff at energies in the range between $200 \mathrm{keV}$ and $\sim 10 \mathrm{MeV}$. In the same figure we reported also the upper limits of 
Mignani et al. (1989) for the optical nebular flux which are in satisfactory agreement with the low frequency extrapolation of the inner plerion spectrum.

\section{Discussion}

The broadband X-ray spectrum of the Vela PWN derived from the combined analysis of BeppoSAX and XMM-Newton data, together with the information from high resolution images of Chandra (Pavlov et al. 2001a,b, 2003), provided a more complete detailed picture of the spectrum and the structure of this source useful for the understanding of the main physical processes responsible for the energy transfer and radiation.

We can distinguish two main regions where $\mathrm{X}$ rays are emitted: a central source of high brightness with an angular diameter of $3^{\prime}-4^{\prime}$ surrounded by a much fainter region extending mainly in the SE and SW sector up to distances of about $12^{\prime}-15^{\prime}$, corresponding to a distance from the pulsar of about $1.2 \mathrm{pc}$. This picture has a good correspondence to the radio images recently obtained by Dodson et al. (2003). These authors report the presence of an extended and highly polarised non-thermal emission out to their field limit of about $6^{\prime}$ from the pulsar. The spatial structure is mainly elongated in the NE-SW direction, but towards the NE it is limited by a quite sharp boundary, not detected in the other direction. Furthermore, the images of Dodson et al. (2003) suggest the presence of another extended component in the SE direction at distances larger than $\sim 6^{\prime}$. Likely, these two outer components are the radio counterparts of the extended X-ray emission detected in the MOS and MECS images.

Our spectral analysis confirms that the central region has a harder spectrum than the outer one, with a photon index of about 1.6 , but possibly close to 1.5 as indicated by the MOS spectra with the smallest extraction radius. Such a hard spectrum was also observed in the high resolution X-ray images obtained by Pavlov et al. (2003) with the Chandra observatory. The photon index from MOS data in the outer region is indeed close to 2 .

The spectrum at energies higher than $\sim 15 \mathrm{keV}$ is well fitted by a single power law with a photon index 2 . A possible interpretation of these results is that the emission from the extended region is described by such a spectrum, while that of the central PWN must show a break at $\sim 12 \mathrm{keV}$ with a photon index change of about 0.5 .

This behaviour does not confirm the claim of De Jager et al. (1996) about the spectrum of the central compact nebula extending unbroken up to $400 \mathrm{keV}$.

In Fig. 6 we compare our broad band results with the hard X-ray SEDs observed by Birmingham 2 (Willmore et al. 1992) and OSSE-CGRO experiments (De Jager et al. 1996). Our spectrum is well above both these data, however, the discrepancy with the data of Willmore et al. (1992) can be explained by the different source extension taken into account. The data of the Birmingham 2 experiment were obtained modelling the coded mask image with a central compact source plus an extended emission. The SED of Fig. 6 refers to the central source only and when scaling our SED to the flux measured by the MECS with an extraction radius of $4^{\prime}$ (see Table 1) we found a good agreement. At variance, the discrepancy with the spectrum derived from the OSSE-CGRO observation (De Jager et al. 1996) is not simply explained by the source extension and can be due to some systematic effect possibly due to the much wider field of view of this experiment. In particular, when these data are extrapolated from $60 \mathrm{keV}$ to energies of $\sim 1 \mathrm{keV}$ (Sefako \& De Jager 2003), the source X-ray luminosity can be largely underestimated. We computed the isotropic Xray luminosity of the Vela plerion by integrating between 0.1 and $200 \mathrm{keV}$ the broken power law spectrum taken over the largest radius and obtained $L_{\mathrm{X}}=5.5 \times 10^{33} \mathrm{erg} \mathrm{s}^{-1}$. This luminosity is more than a factor of 2 higher than that derived from the best fit OSSE spectrum in the same range. Note that about $45 \%$ of this luminosity is emitted at energies lower than $E_{\mathrm{b}}$.

A comparison of the PDS flux of the Crab in the 20-200 keV range and the corresponding OSSE flux based on Ulmer et al. (1995) also shows a discrepancy of the order of $30 \%$.

Pavlov et al. (2001a) estimated that the 1-10 keV luminosity of the PWN within about $1^{\prime}$ from the pulsar is $6 \times$ $10^{32} \mathrm{erg} \mathrm{s}^{-1}$, in a good agreement with that derived by us from the MOS data for a similar extraction radius (Fig. 3). Such a luminosity corresponds to about $1 / 4$ of the emission from the whole source and implies that the outer nebula emits a relevant fraction of the total X-ray power. This more precise value can be useful to improve the estimates of the magnetization parameter $\sigma$ and the pair multiplicity $M$ of the Vela plerion. We did not develop a complex physical model of the source for this purpose, which is beyond the scope of the present paper. However, one can reasonably expect that the greater luminosity obtained by us would imply a higher number of particles and consequently a higher value of $M$. If confirmed by numerical models, like that by Sefako \& De Jager (2003), this finding can be the reason for the too low value found by these authors.

Furthermore, we found that the X-ray emission from the plerion $L_{X}$ is a factor of the order of $\sim 10^{-3}$ of the pulsar spindown luminosity $\dot{E}=6.7 \times 10^{36} \mathrm{erg} \mathrm{s}^{-1}$, one order of magnitude higher than the value given by Pavlov et al. (2001a).

It is possible to show, using a simple dimensional approach, that the nebular extension measured in the MECS images and used in our analysis (i.e. a radius $12^{\prime}-15^{\prime}$, approximately corresponding to $1.0-1.3 \mathrm{pc}$ ) is compatible with the radiative lifetime of high energy electrons $t_{\text {sy }}$ emitting hard $\mathrm{X}$ rays. According to a general plerion model we assume that electrons are injected at the pulsar wind shock, which for Vela is at an angular distance from the pulsar of 53" (Helfand et al. 2001), and then diffuse throughout the plerion. From the synchrotron theory the radiative lifetime of the electrons which emit photons of energy $E_{\gamma}$ is:

$t_{\mathrm{sy}}=2.15 \times 10^{9}\left(\frac{E_{\gamma}}{1 \mathrm{keV}}\right)^{1 / 2}\left(\frac{B_{\perp}}{10^{-4} \mathrm{G}}\right)^{-3 / 2} \mathrm{~s}$

where $B_{\perp}$ is the average transverse component of the magnetic field. The typical time scale to escape the nebula of radius $R$ (in pc units) can be written as

$t_{\mathrm{e}} \simeq R / v_{\mathrm{d}}=1.03 \times 10^{8} a R \quad \mathrm{~s}$ 
where $1 / a$ is the outward diffusion velocity in units of the speed of light $\left(v_{\mathrm{d}}=c / a\right)$; for example, if $v_{\mathrm{d}}$ is equal to the sound velocity in a relativistic plasma then $a=\sqrt{3}$ (De Jager et al. 1996). The two above equations can be used to estimate the highest energy of the photons emitted by electrons able to reach the outer boundary of the plerion (i.e. $t_{\mathrm{e}} \simeq t_{\mathrm{sy}}$ ) and then we obtain:

$E_{\gamma} \simeq \frac{435}{a^{2}}\left(\frac{R}{1 \mathrm{pc}}\right)^{-2}\left(\frac{B_{\perp}}{10^{-4} \mathrm{G}}\right)^{-3} \mathrm{keV}$.

The typical nebular magnetic field is estimated to be about $10^{-4} \mathrm{G}$ (Sefako \& De Jager 2003; Pavlov et al. 2003) and therefore, for any reasonable choice of $a$, photons of energy of $\sim 100 \mathrm{keV}$ can be efficiently radiated up to distances of about $1 \mathrm{pc}$ from the wind shock region without need for a further acceleration. Note that these relations imply a maximum Lorentz factor for the electrons of $\sim 3 \times 10^{8}$, as also estimated in the above quoted papers.

An interesting consequence of Eq. (5) is that photons with energy of $\sim 10 \mathrm{MeV}$ should be emitted from regions much closer to the wind shock and therefore the outer nebula spectrum should show a further steepening at energies higher than a few hundred keV. The occurrence of such a high energy break could be detected by the study of the DC component of the Vela pulsar with the present (INTEGRAL) and the next generation of high sensitivity low-energy $\gamma$ ray telescopes.

Acknowledgements. We are grateful to the referee O. De Jager for his comments which improved our paper. We are also indebted to Bruno Sacco for useful discussions. This work has been partially supported by INAF.

\section{References}

Bocchino, F., Maggio, A., \& Sciortino, S. 1999, A\&A, 342, 839 Boella, G., Butler, R. C., Perola, G. C., et al. 1997, A\&AS, 122, 299 Caraveo, P., De Luca, A., Mignani, R. P., \& Bignami, G. F. 2001, ApJ, 561,930
D’Acri, F., de Grandi, S., \& Molendi, S. 1998, in The Active X-ray Sky: Results from BeppoSAX and RXTE, ed. L. Scarsi, H. Bradt, P. Giommi, \& F. Fiore, Nucl. Phys. B, Proc. Suppl., 69, 581

De Luca, A., \& Molendi, S. 2004, A\&A, 419, 837

De Jager, O. C., Harding, A. K., \& Strickman, M. S. 1996, ApJ, 460, 729

Dodson, R., Legge, D., Reynolds, J. E., \& McCulloch, P. M. 2003, ApJ, 596, 1137

Fiore, F., Guainazzi, M., \& Grandi, P. 1999, in Cookbook for BeppoSAX NFI Spectral Analysis, BeppoSAX Science Data Center, version 1.2

Helfand, D. J., Gotthelf, E. V., \& Halpern, J. P. 2001, ApJ, 556, 380

Kanbach, G., Arzoumanian, Z., Bertsch, D. L., et al. 1994, A\&A, 289 , 855

Kargaltsev, O. Y., \& Pavlov, G. 2003, in Young Neutron Stars and Their Environments, ed. F. Camilo, \& B. M. Gaensler, IAU Symp., 218, 195

Kennell, C. F., \& Coroniti, F. V. 1984, ApJ, 283, 694

Kirsh, M. G. F., Altieri, B., et al. 2004, XMM Newton (cross) calibration, XMM-SOC-CAL-TN-0055

Marty, P. B., Kneib, J.-P., Sadat, R., Ebeling, H., \& Smail, I. 2003, Proc. SPIE., 4851, 208

Mignani, R., De Luca, A., Kargaltsev, O., et al. 2003, ApJ, 594, 419

Molendi, S. 1998, in The Active X-ray Sky: Results from BeppoSAX and RXTE, ed. L. Scarsi, H. Bradt, P. Giommi, \& F. Fiore, Nucl. Phys. B, Proc. Suppl., 69, 563

Mori, K., Hailey, C. J., Paerels, F., \& Zane, S. 2004, Adv. Sp. Res., 33, 503

Pavlov, G. G., Kargaltsev, O. Y., Sanwal, D., \& Garmire, G. P. 2001a, ApJ, 554, L189

Pavlov, G. G., Zavlin, V. E., Sanwal, D., Burwitz, V., \& Garmire, G. P. 2001b, ApJ, 552, L129

Pavlov, G. G., Teter, M. A., de Jager, \& Ocker, C. 2003, ApJ, 591, 1157

Sefako, R. R., \& De Jager, O. C. 2003, ApJ, 593, 1013

Ulmer, M. P., Matz, S. M., Grabelsky, D. A., et al. 1995, ApJ, 448, 356

Weiler, K. W., \& Panagia, N. 1978, A\&A, 70, 419

Willmore, A. P., Eyles, C. J., Skinner, G. K., \& Watt, M. P. 1992, MNRAS, 254, 139 